\title{
OPERATOR-VALUED BERGMAN INNER FUNCTIONS AS TRANSFER FUNCTIONS
}

\author{
A. OLOFSSON
}

\begin{abstract}
An explicit construction characterizing the operator-valued Bergman inner functions is given for a class of vector-valued standard weighted Bergman spaces in the unit disk. These operator-valued Bergman inner functions act as contractive multipliers from the Hardy space into the associated Bergman space, and they have a natural interpretation as transfer functions for a related class of discrete time linear systems. This points to a new interaction between the fields of invariant subspace theory and mathematical systems theory.
\end{abstract}

Let $\mathcal{U}, \mathcal{X}$, and $\mathcal{Y}$ be general not necessarily separable complex Hilbert spaces, and let

$$
A \in \mathcal{L}(\mathcal{X}), \quad B \in \mathcal{L}(\mathcal{U}, \mathcal{X}), \quad C \in \mathcal{L}(\mathcal{X}, \mathcal{Y}), \quad \text { and } \quad D \in \mathcal{L}(\mathcal{U}, \mathcal{Y})
$$

be bounded linear operators. Let $n \geq 1$ be an integer. We shall consider operator-valued analytic functions of the form

$$
W(z)=D+z C\left(\sum_{k=1}^{n}(I-z A)^{-k}\right) B .
$$

Notice that the function $W$ defined by (0.1) is analytic for $z$ close to the origin and that $W(z)$ is an operator in $\mathcal{L}(\mathcal{U}, \mathcal{Y})$.

The operator-valued analytic functions of the form (0.1) admit a natural interpretation as transfer functions for a related class of discrete time linear systems. Consider the discrete time linear system generated by the system of recurrence relations

$$
\left\{\begin{array}{l}
x_{k+1}=A x_{k}+B u_{k}+\sum_{j=0}^{k}\left(\begin{array}{c}
k-j+n-1 \\
k-j+1
\end{array}\right) A^{k-j} B u_{j}, \quad x_{0}=0, \\
y_{k}=C x_{k}+D u_{k},
\end{array}\right.
$$

and set

$$
u(z)=\sum_{k \geq 0} u_{k} z^{k}, \quad y(z)=\sum_{k \geq 0} y_{k} z^{k} .
$$

Then the function $W$ given by (0.1) is the transfer function for system (0.2), which means that $y(z)=W(z) u(z)$ (see Theorem 1.1).

The notation here is adapted from mathematical systems theory. The space $\mathcal{U}$ is the input space, the space $\mathcal{Y}$ is the output space, and the space $\mathcal{X}$ is the state space of system (0.2). The operator $A \in \mathcal{L}(\mathcal{X})$ is called the state space operator, the operator $B \in \mathcal{L}(\mathcal{U}, \mathcal{X})$ is called the input operator, the operator $C \in \mathcal{L}(\mathcal{X}, \mathcal{Y})$ is called the output operator and the operator $D \in \mathcal{L}(\mathcal{U}, \mathcal{Y})$ is called the feedthrough operator for system (0.2).

2000 Mathematics Subject Classification. Primary 47A48; Secondary 47A15.

Key words and phrases. Bergman inner function, transfer function, $n$-hypercontraction, wandering subspace, standard weighted Bergman space, discrete time linear system.

Supported by the M.E.N.R.T. (France) and the G. S. Magnuson's Fund of the Royal Swedish Academy of Sciences.

(C)2008 American Mathematical Society 
System (0.2) can be thought of as a machine that, given an input sequence $\left\{u_{k}\right\}_{k \geq 0}$ in $\mathcal{U}$, produces an output sequence $\left\{y_{k}\right\}_{k \geq 0}$ in $\mathcal{Y}$ by the recurrence relations (0.2). Initially the machine is at rest, and at time $k \geq 0$ the state of the machine shifts from $x_{k}$ to $x_{k+1}$.

We remark that for $n=1$, system (0.2) reduces to the standard type of discrete time linear system

$$
\left\{\begin{array}{l}
x_{k+1}=A x_{k}+B u_{k}, \quad x_{0}=0, \\
y_{k}=C x_{k}+D u_{k},
\end{array}\right.
$$

which was studied by Helton in 13 and by others. The novelty of system (0.2) for $n \geq 2$ is that the state $x_{k+1}$ depends on the previous state $x_{k}$ and on all of the previous inputs $\left\{u_{j}\right\}_{j=0}^{k}$.

We shall need a class of vector-valued standard weighted Bergman spaces that we now proceed to define. Let $n \geq 1$ be an integer, and let $\mathcal{Y}$ be a general not necessarily separable Hilbert space. We denote by $A_{n}(\mathcal{Y})$ the Hilbert space of all $\mathcal{Y}$-valued analytic functions

$$
y(z)=\sum_{k \geq 0} y_{k} z^{k}, \quad z \in \mathbb{D},
$$

in the unit disk $\mathbb{D}$ with finite norm

$$
\|y\|_{A_{n}}^{2}=\sum_{k \geq 0}\left\|y_{k}\right\|^{2} \mu_{n ; k},
$$

where $\mu_{n ; k}=1 /\left(\begin{array}{c}k+n-1 \\ k\end{array}\right)$ for $k \geq 0$. Here the Taylor coefficients $y_{k}$ in (0.4) are elements of $\mathcal{Y}$. The shift operator $S_{n}$ on the space $A_{n}(\mathcal{Y})$ is the operator defined by

$$
\left(S_{n} y\right)(z)=z y(z)=\sum_{k \geq 1} y_{k-1} z^{k}, \quad z \in \mathbb{D},
$$

for $y \in A_{n}(\mathcal{Y})$ given by (0.4). It is easily seen that the shift operator $S_{n}$ is bounded on $A_{n}(\mathcal{Y})$ and has norm equal to 1 (the weight sequence $\left\{\mu_{n ; k}\right\}_{k \geq 0}$ is monotone decreasing and the quotient $\mu_{n ; k+1} / \mu_{n ; k}$ tends to 1 as $\left.k \rightarrow \infty\right)$.

We mention that the weight sequence $\left\{\mu_{n ; k}\right\}_{k \geq 0}$ is a sequence of moments of a radial measure $d \mu_{n}$ on the closed unit disk in the sense that

$$
\mu_{n ; k}=\int_{\overline{\mathbb{D}}}|z|^{2 k} d \mu_{n}(z)=1 /\left(\begin{array}{c}
k+n-1 \\
k
\end{array}\right), \quad k \geq 0,
$$

and the norm of $A_{n}(\mathcal{Y})$ can also be expressed as

$$
\|y\|_{A_{n}}^{2}=\lim _{r \rightarrow 1} \int_{\overline{\mathbb{D}}}\|y(r z)\|^{2} d \mu_{n}(z), \quad y \in A_{n}(\mathcal{Y}) .
$$

The measure $d \mu_{1}$ is the normalized Lebesgue arc length measure on the unit circle $\mathbb{T}=$ $\partial \mathbb{D}$, and for $n \geq 2$, the measure $d \mu_{n}$ is the weighted area measure given by

$$
d \mu_{n}(z)=(n-1)\left(1-|z|^{2}\right)^{n-2} d A(z), \quad z \in \mathbb{D},
$$

where $d A$ is the usual planar Lebesgue area measure normalized so that the unit disk $\mathbb{D}$ is of unit area (see [12, Subsection 1.1]).

A closed subspace $\mathcal{E}$ of $A_{n}(\mathcal{Y})$ is called a wandering subspace if

$$
\mathcal{E} \perp S_{n}^{k}(\mathcal{E}) \text { for } k \geq 1 .
$$

A shift invariant subspace of $A_{n}(\mathcal{Y})$ is a closed subspace $\mathcal{I}$ of $A_{n}(\mathcal{Y})$ such that $S_{n}(\mathcal{I}) \subset \mathcal{I}$. Wandering subspaces arise from shift invariant subspaces in a natural way. If $\mathcal{I}$ is a shift invariant subspace of $A_{n}(\mathcal{Y})$, then the subspace

$$
\mathcal{E}=\mathcal{I} \ominus S_{n}(\mathcal{I})
$$


is a wandering subspace of $A_{n}(\mathcal{Y})$ called the wandering subspace for $\mathcal{I}$. In favorable cases, a shift invariant subspace $\mathcal{I}$ of $A_{n}(\mathcal{Y})$ can be reconstructed by its wandering subspace $\mathcal{E}$ in the sense that

$$
\mathcal{I}=[\mathcal{E}]=\bigvee_{k \geq 0} S_{n}^{k}(\mathcal{E})
$$

By now, there are well-known results by Halmos [10, Aleman, Richter, and Sundberg [3] and Shimorin [18, 19] establishing (0.5) for a general shift invariant subspace $\mathcal{I}$ of $A_{n}(\mathcal{Y})$ for the values $n=1,2,3$; see also [14 for some related summability results. Approximation results of this type clearly point to the need for a better understanding of the structure of wandering subspaces in the Bergman spaces.

Definition 0.1. Let $W$ be an $\mathcal{L}(\mathcal{U}, \mathcal{Y})$-valued analytic function in the unit disk $\mathbb{D}$. The function $W$ is called an operator-valued Bergman inner function for $A_{n}(\mathcal{Y})$ if it has the following two properties:

(1) For every $u \in \mathcal{U}$ the function $W u: z \mapsto W(z) u$ is in $A_{n}(\mathcal{Y})$ and $\|W u\|_{A_{n}}^{2}=\|u\|^{2}$.

(2) $W u \perp S_{n}^{k}(W u)$ in $A_{n}(\mathcal{Y})$ for every $k \geq 1$ and $u \in \mathcal{U}$.

Note that, by property (11) of Bergman inner functions, the range

$$
W(\mathcal{U})=\left\{W u \in A_{n}(\mathcal{Y}): u \in \mathcal{U}\right\}
$$

is a closed subspace of $A_{n}(\mathcal{Y})$; this subspace is isometrically identified with $\mathcal{U}$. By a polarization argument, from property (2) we deduce that $W u \perp S_{n}^{k}(W v)$ in $A_{n}(\mathcal{Y})$ for all $k \geq 1$ and $u, v \in \mathcal{U}$. This means that the range $W(\mathcal{U})$ is a wandering subspace of $A_{n}(\mathcal{Y})$.

We shall show in this paper that a general wandering subspace $\mathcal{E}$ of $A_{n}(\mathcal{Y})$ can be parameterized by an $\mathcal{L}(\mathcal{U}, \mathcal{Y})$-valued Bergman inner function $W$ for $A_{n}(\mathcal{Y})$ in the sense that

$$
\mathcal{E}=\left\{W u \in A_{n}(\mathcal{Y}): u \in \mathcal{U}\right\}
$$

(see Theorem 4.1). The $\mathcal{L}(\mathcal{U}, \mathcal{Y})$-valued Bergman inner function $W$ arising in (0.6) is uniquely determined up to a unitary movement of the input space $\mathcal{U}$ (see Proposition 4.1).

Examples of Bergman inner functions are provided by operator-valued analytic functions $W$ in $\mathbb{D}$ of the form (0.1), and conversely, a general operator-valued Bergman inner function can be realized as an operator-valued analytic function $W$ in $\mathbb{D}$ of the form (0.1). These results provide a complete explicit description of the $\mathcal{L}(\mathcal{U}, \mathcal{Y})$-valued Bergman inner functions for $A_{n}(\mathcal{Y})$ involving operator (in)equalities. In this way we obtain a new interpretation of operator-valued Bergman inner functions as transfer functions for a class of systems of the form (0.2). To describe these results, we need some more preparation.

Let $n \geq 1$ be an integer. Following Agler [1, 2, we call an operator $A \in \mathcal{L}(\mathcal{X})$ an $n$-hypercontraction if

$$
\sum_{k=0}^{m}(-1)^{k}\left(\begin{array}{c}
m \\
k
\end{array}\right) A^{* k} A^{k} \geq 0 \text { in } \mathcal{L}(\mathcal{X})
$$

for $1 \leq m \leq n$. Associated with an $n$-hypercontraction $A \in \mathcal{L}(\mathcal{X})$ we have the operators

$$
D_{m, A}=\left(\sum_{k=0}^{m}(-1)^{k}\left(\begin{array}{c}
m \\
k
\end{array}\right) A^{* k} A^{k}\right)^{1 / 2} \text { in } \mathcal{L}(\mathcal{X})
$$

for $1 \leq m \leq n$, where the positive square root is used. The space $\mathcal{D}_{m, A}$ is the closure in $\mathcal{X}$ of the range of the operator $D_{m, A}$, that is, $\mathcal{D}_{m, A}=\overline{D_{m, A}(\mathcal{X})}$. For a contraction operator $A \in \mathcal{L}(\mathcal{X}, \mathcal{Y})$ we also use the standard notation $D_{A}=\left(I-A^{*} A\right)^{1 / 2}$ and $\mathcal{D}_{A}=\overline{D_{A}(\mathcal{X})}$ for the defect operator and the defect space, respectively. 
We also need some operator model theory for $n$-hypercontractions. Recall that an operator $A \in \mathcal{L}(\mathcal{X})$ is said to be of class $C_{0}$. if $\lim _{k \rightarrow \infty} A^{k}=0$ in the strong operator topology, meaning that $\lim _{k \rightarrow \infty} A^{k} x=0$ in $\mathcal{X}$ for every $x \in \mathcal{X}$ (see [20, II.4]). Let $A \in \mathcal{L}(\mathcal{X})$ be an $n$-hypercontraction of class $C_{0}$.. For $x \in \mathcal{X}$, we define a $\mathcal{D}_{n, A}$-valued analytic function $V_{n} x$ in the unit disk $\mathbb{D}$ by the formula

$$
\left(V_{n} x\right)(z)=D_{n, A}(I-z A)^{-n} x=\sum_{k \geq 0} \frac{1}{\mu_{n ; k}}\left(D_{n, A} A^{k} x\right) z^{k}, \quad z \in \mathbb{D} .
$$

In earlier work we have shown that the map $V_{n}: x \mapsto V_{n} x$ is an isometry

$$
V_{n}: \mathcal{H} \rightarrow A_{n}\left(\mathcal{D}_{n, A}\right)
$$

of $\mathcal{H}$ into $A_{n}\left(\mathcal{D}_{n, A}\right)$ such that

$$
V_{n} A=S_{n}^{*} V_{n}
$$

In this way, an $n$-hypercontraction $A \in \mathcal{L}(\mathcal{X})$ in the class $C_{0}$. is naturally modeled as part (restriction to an invariant subspace) of the adjoint shift operator $S_{n}^{*}$ on $A_{n}\left(\mathcal{D}_{n, A}\right)$ by the map $V_{n}$, which puts the earlier result of Agler [1, 2] in a more explicit form. We have also shown that this model of an $n$-hypercontraction of class $C_{0}$. as part of an adjoint shift $S_{n}^{*}$ is unique up to a movement of the coefficient space $\mathcal{D}_{n, A}$. The proofs of these results can be found in [15, $\S 6$ and $\S 7]$. Here we also mention the recent related publications in a multivariable context by Ambrozie, Engliš, and Müller [4] and Arazy and Engliš [5].

Let $A \in \mathcal{L}(\mathcal{X})$ be an $n$-hypercontraction. We shall consider bounded linear operators

$$
B \in \mathcal{L}(\mathcal{U}, \mathcal{X}), \quad C \in \mathcal{L}(\mathcal{X}, \mathcal{Y}), \text { and } D \in \mathcal{L}(\mathcal{U}, \mathcal{Y})
$$

such that

$$
\begin{aligned}
& C^{*} C=\sum_{k=0}^{n}(-1)^{k}\left(\begin{array}{l}
n \\
k
\end{array}\right) A^{* k} A^{k} \text { in } \mathcal{L}(\mathcal{X}), \\
& D^{*} C+B^{*}\left(\sum_{k=0}^{n-1}(-1)^{k}\left(\begin{array}{c}
n \\
k+1
\end{array}\right) A^{* k} A^{k}\right) A=0 \text { in } \mathcal{L}(\mathcal{X}, \mathcal{U}), \text { and } \\
& D^{*} D+B^{*}\left(\sum_{k=0}^{n-1}(-1)^{k}\left(\begin{array}{c}
n \\
k+1
\end{array}\right) A^{* k} A^{k}\right) B=I \text { in } \mathcal{L}(\mathcal{U}) .
\end{aligned}
$$

We shall show that if $(A, B, C, D)$ is such a collection of operators and the $n$-hypercontraction $A \in \mathcal{L}(\mathcal{X})$ is also in the class $C_{0}$, then the function $W$ given by (0.1) is an $\mathcal{L}(\mathcal{U}, \mathcal{Y})$-valued Bergman inner function for $A_{n}(\mathcal{Y})$ in the sense of Definition 0.1 (see Theorem 2.1).

Conversely, we show that a general $\mathcal{L}(\mathcal{U}, \mathcal{Y})$-valued Bergman inner function for $A_{n}(\mathcal{Y})$ can be realized as an operator-valued analytic function $W$ of the form (0.1), where $A \in \mathcal{L}(\mathcal{X})$ is an $n$-hypercontraction of class $C_{0}$. and the collection $(A, B, C, D)$ of operators satisfies (0.7)-(0.9) (see Theorem 4.2). These results (Theorems 2.1 and 4.2) provide a complete explicit description of the operator-valued Bergman inner functions (see Definition (0.1) involving identities (0.7)-(0.9) and the notion of an $n$-hypercontraction of class $C_{0}$.

As soon as we know that operator-valued Bergman inner functions for $A_{n}(\mathcal{Y})$ correspond to transfer functions for a class of systems of the form (0.2), the problem of finding efficient realizations of this kind arises. This is similar to what is known as the realization problem in mathematical systems theory, and we discuss this matter in $\$ 5$ in this paper. A system of the form (0.2) in which $A \in \mathcal{L}(\mathcal{X})$ is an $n$-hypercontraction of class 
$C_{0}$. and the quadruple of operators $(A, B, C, D)$ satisfies (0.7)-(0.9) is always approximately observable (see Theorem 5.1), but in general is not approximately controllable (see Theorems 5.2 and 5.3). At least under an additional condition on the operators $A$ and $B$ the property of approximate controllability for such a system (0.2) seems to be related to the property that the subspace $V_{n}(\mathcal{X})$ of $A_{n}\left(\mathcal{D}_{n, A}\right)$ contains no nonzero shift invariant subspace (see Theorem [5.3). However, given a realization (0.2) of a Bergman inner function, we can always restrict the state space to the approximately controllable subspace

$$
\mathcal{X}_{c}=\bigvee_{k \geq 0} A^{k} B(\mathcal{U})
$$

of $\mathcal{X}$ to make the system (0.2) minimal.

Identities (0.7)-(0.9) can be interpreted in terms of an associated block operator matrix. Let $A \in \mathcal{L}(\mathcal{X})$ be an $n$-hypercontraction. We denote by $\mathcal{X}_{n}$ the space $\mathcal{X}$ equipped with the equivalent norm

$$
\|x\|_{n}^{2}=\sum_{k=0}^{n-1}(-1)^{k}\left(\begin{array}{c}
n \\
k+1
\end{array}\right)\left\|A^{k} x\right\|^{2}=\|x\|^{2}+\sum_{k=1}^{n-1}\left\|D_{k, A} x\right\|^{2}, \quad x \in \mathcal{X}_{n} .
$$

The fact that these two expressions for the norm $\|\cdot\|_{n}$ are equal follows by [16, Lemma 3.1]. Observe that $\mathcal{X}_{1}=\mathcal{X}$. We denote by $I_{n}: \mathcal{X} \rightarrow \mathcal{X}_{n}$ the inclusion map of $\mathcal{X}$ into $\mathcal{X}_{n}$ defined by $I_{n} x=x$ for $x \in \mathcal{X}$. Identities (0.7)-(0.9) can then be restated by saying that the block operator matrix

$$
\theta_{n}=\left[\begin{array}{cc}
A_{n} & B_{n} \\
C & D
\end{array}\right]=\left[\begin{array}{cc}
I_{n} A & I_{n} B \\
C & D
\end{array}\right]: \mathcal{X} \oplus \mathcal{U} \rightarrow \mathcal{X}_{n} \oplus \mathcal{Y}
$$

is an isometry of $\mathcal{X} \oplus \mathcal{U}$ into $\mathcal{X}_{n} \oplus \mathcal{Y}$ (see Theorem 3.1). Note that the block operator matrix

$$
\theta_{1}=\left[\begin{array}{ll}
A & B \\
C & D
\end{array}\right]: \mathcal{X} \oplus \mathcal{U} \rightarrow \mathcal{X} \oplus \mathcal{Y}
$$

is the so-called system matrix for system (0.3), and recall the terminology that system (0.3) is said to be isometric if the system matrix $\theta_{1}$ is an isometry of $\mathcal{X} \oplus \mathcal{U}$ into $\mathcal{X} \oplus \mathcal{Y}$.

Let $A \in \mathcal{L}(\mathcal{X})$ be an $n$-hypercontraction. There is a natural choice of operators $B$, $C$, and $D$ that makes the block operator matrix $\theta_{n}$ a unitary operator. It turns out that the operator $A_{n}=I_{n} A$ in $\mathcal{L}\left(\mathcal{X}, \mathcal{X}_{n}\right)$ is a contraction operator with defect operator $D_{A_{n}}=D_{n, A}$ in $\mathcal{L}(\mathcal{X})$ (see Lemma 3.2). By a well-known construction going back to Halmos [9, this allows us to conclude that the block operator matrix

$$
\left[\begin{array}{cc}
A_{n} & B_{n} \\
C & D
\end{array}\right]=\left[\begin{array}{cc}
I_{n} A & D_{A_{n}^{*}} \\
D_{n, A} & -A_{n}^{*}
\end{array}\right]: \mathcal{X} \oplus \mathcal{D}_{A_{n}^{*}} \rightarrow \mathcal{X}_{n} \oplus \mathcal{D}_{n, A}
$$

is a unitary operator of $\mathcal{X} \oplus \mathcal{D}_{A_{n}^{*}}$ onto $\mathcal{X}_{n} \oplus \mathcal{D}_{n, A}$ (see Theorem 3.2). The operator-valued analytic function $W$ of the form (0.1) corresponding to this quadruple $(A, B, C, D)$ of operators is given by

$$
W_{n, A}(z)=\left.\left[-A_{n}^{*}+z D_{n, A}\left(\sum_{k=1}^{n}(I-z A)^{-k}\right) I_{n}^{-1} D_{A_{n}^{*}}\right]\right|_{\mathcal{D}_{A_{n}^{*}}}, \quad z \in \mathbb{D} .
$$

The function $W_{n, A}$ is analytic in $\mathbb{D}$ because $\|A\| \leq 1$ and $W_{n, A}(z)$ is an operator in $\mathcal{L}\left(\mathcal{D}_{A_{n}^{*}}, \mathcal{D}_{n, A}\right)$.

We remark that in the case where $n=1$ and $A \in \mathcal{L}(\mathcal{X})$ is a contraction operator, this construction gives the so-called characteristic operator function

$$
W_{A}(z)=W_{1, A}(z)=\left.\left[-A^{*}+z D_{A}(I-z A)^{-1} D_{A^{*}}\right]\right|_{\mathcal{D}_{A^{*}}}, \quad z \in \mathbb{D},
$$


studied by Sz.-Nagy and Foiaş [20, Chapter VI]; the values $W_{A}(z)$ are operators in $\mathcal{L}\left(\mathcal{D}_{A^{*}}, \mathcal{D}_{A}\right)$. The characteristic operator function $W_{A}$ is often thought of as the transfer function for the unitary system (0.3) with system matrix

$$
\left[\begin{array}{cc}
A & B \\
C & D
\end{array}\right]=\left[\begin{array}{cc}
A & D_{A^{*}} \\
D_{A} & -A^{*}
\end{array}\right]: \mathcal{X} \oplus \mathcal{D}_{A^{*}} \rightarrow \mathcal{X} \oplus \mathcal{D}_{A}
$$

(see [8, XXVIII.4]).

We also show that the operator-valued Bergman inner functions act as contractive multipliers from the Hardy space $A_{1}(\mathcal{U})$ into the Bergman space $A_{n}(\mathcal{Y})$ (see Theorem 6.1). This multiplier property can be restated as a norm bound for the input-output map for the corresponding system of the form (0.2) (see Corollary 6.2). We mention that in the scalar case the contractive multiplier property of Bergman inner functions goes back to Hedenmalm [1].

\section{§1. System-theoretic interpretation}

Our purpose in this section is to interpret an operator-valued analytic function of the form (0.1) as the transfer function for the discrete time linear system (0.2). This interpretation is provided by Theorem 1.1 below.

We start with a preparatory remark. Let $m \geq 1$ be an integer, and consider the standard power series expansion

$$
\frac{1}{(1-z)^{m}}=\sum_{k \geq 0} \frac{1}{\mu_{m ; k}} z^{k}, \quad z \in \mathbb{D}
$$

where $1 / \mu_{m ; k}=\left(\begin{array}{c}k+m-1 \\ k\end{array}\right)$ for $k \geq 0$. Computing the backward shift of this power series, we arrive at the formula

$$
\sum_{k \geq 0} \frac{1}{\mu_{m ; k+1}} z^{k}=\frac{1}{z}\left(\frac{1}{(1-z)^{m}}-1\right)=\sum_{k=1}^{m} \frac{1}{(1-z)^{k}}, \quad z \in \mathbb{D}
$$

where the last identity is elementary.

An operator-valued analytic function of the form (0.1) can be generated by a system of recurrence relations as follows.

Theorem 1.1. Suppose the sequences $\left\{u_{k}\right\}_{k \geq 0},\left\{x_{k}\right\}_{k \geq 0}$, and $\left\{y_{k}\right\}_{k \geq 0}$ in the spaces $\mathcal{U}$, $\mathcal{X}$, and $\mathcal{Y}$, respectively, are obtained from the system of recurrence relations

$$
\begin{aligned}
x_{k+1} & =A x_{k}+B u_{k}+\sum_{j=0}^{k}\left(\begin{array}{c}
k-j+n-1 \\
k-j+1
\end{array}\right) A^{k-j} B u_{j}, \quad x_{0}=0, \\
y_{k} & =C x_{k}+D u_{k} .
\end{aligned}
$$

Then $y(z)=W(z) u(z)$, where the function $W$ is given by (0.1) and

$$
u(z)=\sum_{k \geq 0} u_{k} z^{k}, \quad y(z)=\sum_{k \geq 0} y_{k} z^{k} .
$$

Proof. Put $x(z)=\sum_{k \geq 0} x_{k} z^{k}$. By the first of the recurrence relations, we have

$$
x(z) / z=A x(z)+B u(z)+\left(\sum_{k \geq 0}\left(\begin{array}{c}
k+n-1 \\
k+1
\end{array}\right) z^{k} A^{k}\right) B u(z) .
$$


Recall the power series expansion (1.1). We set $m=n-1$ and substitute the operator $z A$ for $z$ in (1.1) to conclude that

$$
\sum_{k \geq 0}\left(\begin{array}{c}
k+n-1 \\
k+1
\end{array}\right) z^{k} A^{k}=\sum_{k=1}^{n-1}(I-z A)^{-k} .
$$

Next, we substitute this identity in (1.2) and solve for $x(z)$, obtaining

$$
x(z)=z\left(\sum_{k=1}^{n}(I-z A)^{-k}\right) B u(z) .
$$

By the last of the recurrence relations we have

$$
y(z)=C x(z)+D u(z)=z C\left(\sum_{k=1}^{n}(I-z A)^{-k}\right) B u(z)+D u(z)=W(z) u(z),
$$

where the function $W$ is given by (0.1). This completes the proof of the theorem.

Remark 1.1. We remark that the function $x(z)=\sum_{k \geq 0} x_{k} z^{k}$ is given by

$$
x(z)=z\left(\sum_{k=1}^{n}(I-z A)^{-k}\right) B u(z) .
$$

In fact, this is the content of the first of the recurrence relations in Theorem 1.1,

\section{§2. Construction of Bergman inner functions}

In this section we give a construction of Bergman inner functions of the form (0.1) using identities (0.7)-(0.9). This construction is provided by Theorem 2.1 below.

First, we compute the power series expansion of the function $W$ given by (0.1). Recall the power series expansion (1.1). We set $m=n$ and substitute the operator $z A$ for $z$ in (1.1) to obtain

$$
\sum_{k=1}^{n}(1-z A)^{-k}=\sum_{k \geq 0} \frac{1}{\mu_{n ; k+1}} A^{k} z^{k}
$$

This makes obvious the power series expansion

$$
W(z)=D+\sum_{k \geq 1} \frac{1}{\mu_{n ; k}}\left(C A^{k-1} B\right) z^{k}
$$

of the function $W$.

For easy reference, we record the following lemma.

Lemma 2.1. Let $A \in \mathcal{L}(\mathcal{X})$ be an n-hypercontraction of class $C_{0}$.. Then

$$
\sum_{k=0}^{n-1}(-1)^{k}\left(\begin{array}{c}
n \\
k+1
\end{array}\right)\left\|A^{k} x\right\|^{2}=\sum_{k \geq 0} \frac{1}{\mu_{n ; k+1}}\left\|D_{n, A} A^{k} x\right\|^{2}, \quad x \in \mathcal{X} .
$$

Proof. See [16, Lemma 2.3]. The proof involves the operator model theory for $n$-hypercontractions; see [15, §7]. We omit the details.

Theorem 2.1. Let $A \in \mathcal{L}(\mathcal{X})$ be an n-hypercontraction of class $C_{0}$, and let

$$
B \in \mathcal{L}(\mathcal{U}, \mathcal{X}), \quad C \in \mathcal{L}(\mathcal{X}, \mathcal{Y}), \quad \text { and } \quad D \in \mathcal{L}(\mathcal{U}, \mathcal{Y})
$$

be operators satisfying (0.7), (0.8), and (0.9). Then the function $W$ given by (0.1) is an $\mathcal{L}(\mathcal{U}, \mathcal{Y})$-valued Bergman inner function for $A_{n}(\mathcal{Y})$. 
Proof. First, we show that $\|W u\|_{A_{n}}^{2}=\|u\|^{2}$ for $u \in \mathcal{U}$ (property (1) of Bergman inner functions). By the power series expansion (2.1), we have

$$
\|W u\|_{A_{n}}^{2}=\|D u\|^{2}+\sum_{k \geq 1} \frac{1}{\mu_{n ; k}}\left\|C A^{k-1} B u\right\|^{2} .
$$

Notice that $\left\|C A^{k-1} B u\right\|^{2}=\left\|D_{n, A} A^{k-1} B u\right\|^{2}$ by (0.7). Using Lemma 2.1, we conclude that

$$
\begin{aligned}
\|W u\|_{A_{n}}^{2} & =\|D u\|^{2}+\sum_{k \geq 1} \frac{1}{\mu_{n ; k}}\left\|D_{n, A} A^{k-1} B u\right\|^{2} \\
& =\|D u\|^{2}+\sum_{k=0}^{n-1}(-1)^{k}\left(\begin{array}{c}
n \\
k+1
\end{array}\right)\left\|A^{k} B u\right\|^{2} \\
& =\left\langle\left(D^{*} D+B^{*}\left(\sum_{k=0}^{n-1}(-1)^{k}\left(\begin{array}{c}
n \\
k+1
\end{array}\right) A^{* k} A^{k}\right) B\right) u, u\right\rangle=\|u\|^{2},
\end{aligned}
$$

where the last identity follows from (0.9).

Next we show that $W u \perp S_{n}^{k}(W u)$ for $u \in \mathcal{U}$ and $k \geq 1$ (property (2) of Bergman inner functions). Observe that

$$
\left(S_{n}^{k} W u\right)(z)=(D u) z^{k}+\sum_{j \geq k+1} \frac{1}{\mu_{n ; j-k}}\left(C A^{j-k-1} B u\right) z^{j}, \quad z \in \mathbb{D},
$$

by (2.1). We have

$$
\begin{aligned}
\left\langle W u, S_{n}^{k} W u\right\rangle_{A_{n}} & =\left\langle C A^{k-1} B u, D u\right\rangle+\sum_{j \geq k+1} \frac{1}{\mu_{n ; j-k}}\left\langle C A^{j-1} B u, C A^{j-k-1} B u\right\rangle \\
& =\left\langle C A^{k-1} B u, D u\right\rangle+\sum_{j \geq 0} \frac{1}{\mu_{n ; j+1}}\left\langle D_{n, A} A^{j} A^{k} B u, D_{n, A} A^{j} B u\right\rangle,
\end{aligned}
$$

where the second identity follows by (0.7) and a change in the order of summation. A polarization argument and Lemma 2.1 show that

$$
\sum_{j \geq 0} \frac{1}{\mu_{n ; j+1}}\left\langle D_{n, A} A^{j} x_{1}, D_{n, A} A^{j} x_{2}\right\rangle=\sum_{j=0}^{n-1}(-1)^{j}\left(\begin{array}{c}
n \\
j+1
\end{array}\right)\left\langle A^{j} x_{1}, A^{j} x_{2}\right\rangle, \quad x_{1}, x_{2} \in \mathcal{X} .
$$

This allows us to conclude that

$$
\begin{aligned}
\left\langle W u, S_{n}^{k} W u\right\rangle_{A_{n}} & =\left\langle C A^{k-1} B u, D u\right\rangle+\sum_{j=0}^{n-1}(-1)^{j}\left(\begin{array}{c}
n \\
j+1
\end{array}\right)\left\langle A^{j} A^{k} B u, A^{j} B u\right\rangle \\
& =\left\langle\left(D^{*} C+B^{*}\left(\sum_{j=0}^{n-1}(-1)^{j}\left(\begin{array}{c}
n \\
j+1
\end{array}\right) A^{* j} A^{j}\right) A\right) A^{k-1} B u, u\right\rangle=0,
\end{aligned}
$$

where the last identity is a consequence of (0.8). This completes the proof of the theorem.

Remark 2.1. We remark that in Theorem 2.1 it suffices to assume that identities (0.7) and (0.8) are fulfilled when acting on elements of the subspace $\bigvee_{k \geq 0} A^{k} B(\mathcal{U})$ of $\mathcal{X}$. The subspace $\mathcal{X}_{c}=\bigvee_{k \geq 0} A^{k} B(\mathcal{U})$ of $\mathcal{X}$ admits the system-theoretic interpretation of being the approximately controllable subspace of $\mathcal{X}$. 


\section{§3. Block matrix interpretation of identities (0.7)-(0.9)}

In this section we shall interpret the identities $0.7-0.9$ in terms of the associated block operator matrix $\theta_{n}$ in Theorem 3.1. This interpretation allows us to give a canonical example of operators satisfying these identities in Theorem 3.2 below.

Let $A \in \mathcal{L}(\mathcal{X})$ be an $n$-hypercontraction. We shall denote by $\mathcal{X}_{n}$ the space $\mathcal{X}$ equipped with the equivalent norm

$$
\|x\|_{n}^{2}=\sum_{k=0}^{n-1}(-1)^{k}\left(\begin{array}{c}
n \\
k+1
\end{array}\right)\left\|A^{k} x\right\|^{2}=\|x\|^{2}+\sum_{k=1}^{n-1}\left\|D_{k, A} x\right\|^{2}, \quad x \in \mathcal{X}_{n} .
$$

The fact that the two expressions for the norm $\|\cdot\|_{n}$ in (3.1) are equal follows from [16. Lemma 3.1]. Note that the norm $\|\cdot\|_{1}$ coincides with the usual norm of $\mathcal{X}$. We denote by $I_{n}: \mathcal{X} \rightarrow \mathcal{X}_{n}$ the inclusion map of $\mathcal{X}$ into $\mathcal{X}_{n}$ defined by $I_{n} x=x$ for $x \in \mathcal{X}$.

Lemma 3.1. Let $A \in \mathcal{L}(\mathcal{X})$ be an n-hypercontraction, and consider the inclusion map $I_{n}: \mathcal{X} \rightarrow \mathcal{X}_{n}$ defined by $I_{n} x=x$ for $x \in \mathcal{X}$. Then the adjoint operator $I_{n}^{*} \in \mathcal{L}\left(\mathcal{X}_{n}, \mathcal{X}\right)$ acts by the formula

$$
I_{n}^{*} x=\left(\sum_{k=0}^{n-1}(-1)^{k}\left(\begin{array}{c}
n \\
k+1
\end{array}\right) A^{* k} A^{k}\right) x, \quad x \in \mathcal{X}_{n}
$$

Proof. The computation is straightforward; see [16, Lemma 3.2].

Next we interpret (0.7)-(0.9) in terms of a certain associated block operator matrix.

Theorem 3.1. Let $A \in \mathcal{L}(\mathcal{X})$ be an n-hypercontraction, and let $B \in \mathcal{L}(\mathcal{U}, \mathcal{X}), C \in$ $\mathcal{L}(\mathcal{X}, \mathcal{Y})$, and $D \in \mathcal{L}(\mathcal{U}, \mathcal{Y})$ be bounded linear operators. Set $A_{n}=I_{n} A \in \mathcal{L}\left(\mathcal{X}, \mathcal{X}_{n}\right)$ and $B_{n}=I_{n} B \in \mathcal{L}\left(\mathcal{U}, \mathcal{X}_{n}\right)$. Then the block operator matrix

$$
\theta_{n}=\left[\begin{array}{cc}
A_{n} & B_{n} \\
C & D
\end{array}\right]: \mathcal{X} \oplus \mathcal{U} \rightarrow \mathcal{X}_{n} \oplus \mathcal{Y}
$$

is an isometry of $\mathcal{X} \oplus \mathcal{U}$ into $\mathcal{X}_{n} \oplus \mathcal{Y}$ if and only if identities (0.7), (0.8), and (0.9) are fulfilled.

Proof. A computation shows that

$$
\theta_{n}^{*} \theta_{n}=\left[\begin{array}{ll}
A_{n}^{*} A_{n}+C^{*} C & A_{n}^{*} B_{n}+C^{*} D \\
B_{n}^{*} A_{n}+D^{*} C & B_{n}^{*} B_{n}+D^{*} D
\end{array}\right]
$$

By Lemma 3.1, we have

$$
\begin{aligned}
A_{n}^{*} A_{n}+C^{*} C & =A^{*}\left(\sum_{k=0}^{n-1}(-1)^{k}\left(\begin{array}{c}
n \\
k+1
\end{array}\right) A^{* k} A^{k}\right) A+C^{*} C \\
& =I-\sum_{k=0}^{n}(-1)^{k}\left(\begin{array}{l}
n \\
k
\end{array}\right) A^{* k} A^{k}+C^{*} C \text { in } \mathcal{L}(\mathcal{X}) .
\end{aligned}
$$

This shows that the identity $A_{n}^{*} A_{n}+C^{*} C=I$ in $\mathcal{L}(\mathcal{X})$ is equivalent to (0.7).

By a similar argument, we check that the identity $B_{n}^{*} A_{n}+D^{*} C=0$ in $\mathcal{L}(\mathcal{X}, \mathcal{U})$ is equivalent to (0.8), and that the identity $B_{n}^{*} B_{n}+D^{*} D=I$ in $\mathcal{L}(\mathcal{U})$ is equivalent to (0.9). This completes the proof of the theorem.

We recall a few facts about contraction operators. Let $A \in \mathcal{L}(\mathcal{X}, \mathcal{Y})$ be a contraction operator. The defect operator for $A$ is the operator

$$
D_{A}=\left(I-A^{*} A\right)^{1 / 2} \text { in } \mathcal{L}(\mathcal{X})
$$


where the positive square root is used. The defect space $\mathcal{D}_{A}$ for $A$ is the closure in $\mathcal{X}$ of the range of the operator $D_{A}$, that is, $\mathcal{D}_{A}=\overline{D_{A}(\mathcal{X})}$. Note that $A^{*} A+D_{A}^{2}=I$ in $\mathcal{L}(\mathcal{X})$. Similarly, the adjoint operator $A^{*} \in \mathcal{L}(\mathcal{Y}, \mathcal{X})$ has a defect operator

$$
D_{A^{*}}=\left(I-A A^{*}\right)^{1 / 2} \text { in } \mathcal{L}(\mathcal{Y}),
$$

and a defect space $\mathcal{D}_{A^{*}}$ contained in $\mathcal{Y}$. We shall also need the formulas

$$
D_{A^{*}} A=A D_{A} \text { in } \mathcal{L}(\mathcal{X}, \mathcal{Y}) \text { and } D_{A} A^{*}=A^{*} D_{A^{*}} \text { in } \mathcal{L}(\mathcal{Y}, \mathcal{X})
$$

(see [8, XXVII.1] or [9]). A straightforward computation involving formulas (3.2) shows that the block operator matrix

$$
\left[\begin{array}{cc}
A & D_{A^{*}} \\
D_{A} & -A^{*}
\end{array}\right]: \mathcal{X} \oplus \mathcal{D}_{A^{*}} \rightarrow \mathcal{Y} \oplus \mathcal{D}_{A}
$$

is a unitary operator of $\mathcal{X} \oplus \mathcal{D}_{A^{*}}$ onto $\mathcal{Y} \oplus \mathcal{D}_{A}$. The construction of this unitary block operator matrix goes back to Halmos $[9]$.

Now we consider the operator $A_{n}=I_{n} A$ in $\mathcal{L}\left(\mathcal{X}, \mathcal{X}_{n}\right)$ in some more detail.

Lemma 3.2. Let $A \in \mathcal{L}(\mathcal{X})$ be an n-hypercontraction, and consider the operator $A_{n}=$ $I_{n} A$ in $\mathcal{L}\left(\mathcal{X}, \mathcal{X}_{n}\right)$. Then $A_{n}$ is a contraction operator in $\mathcal{L}\left(\mathcal{X}, \mathcal{X}_{n}\right)$ with defect operator and defect space given by

$$
D_{A_{n}}=D_{n, A} \text { in } \mathcal{L}(\mathcal{X}) \text { and } \mathcal{D}_{A_{n}}=\mathcal{D}_{n, A} .
$$

The adjoint operator $A_{n}^{*}$ in $\mathcal{L}\left(\mathcal{X}_{n}, \mathcal{X}\right)$ acts by the formula

$$
A_{n}^{*} x=A^{*}\left(\sum_{k=0}^{n-1}(-1)^{k}\left(\begin{array}{c}
n \\
k+1
\end{array}\right) A^{* k} A^{k}\right) x, \quad x \in \mathcal{X}_{n},
$$

and the action of the defect operator $D_{A_{n}^{*}}$ in $\mathcal{L}\left(\mathcal{X}_{n}\right)$ of $A_{n}^{*}$ is given by

$$
D_{A_{n}^{*}} x=\left(I-A A^{*}\left(\sum_{k=0}^{n-1}(-1)^{k}\left(\begin{array}{c}
n \\
k+1
\end{array}\right) A^{* k} A^{k}\right)\right)^{1 / 2} x, \quad x \in \mathcal{X}_{n},
$$

where the positive square root is computed in $\mathcal{L}\left(\mathcal{X}_{n}\right)$.

Proof. First, we show that $A_{n}$ is a contraction operator in $\mathcal{L}\left(\mathcal{X}, \mathcal{X}_{n}\right)$. We employ the formula

$$
\left\|D_{k+1, A} x\right\|^{2}=\left\|D_{k, A} x\right\|^{2}-\left\|D_{k, A} A x\right\|^{2}, \quad x \in \mathcal{X},
$$

for defect operators $(1 \leq k<n)$. Using this formula, we compute

$$
\begin{aligned}
\left\|A_{n} x\right\|_{n}^{2} & =\|A x\|^{2}+\sum_{k=1}^{n-1}\left(\left\|D_{k, A} x\right\|^{2}-\left\|D_{k+1, A} x\right\|^{2}\right) \\
& =\|A x\|^{2}+\left\|D_{1, A} x\right\|^{2}-\left\|D_{n, A} x\right\|^{2}=\|x\|^{2}-\left\|D_{n, A} x\right\|^{2}, \quad x \in \mathcal{X} .
\end{aligned}
$$

This shows that $A_{n}$ is a contraction operator in $\mathcal{L}\left(\mathcal{X}, \mathcal{X}_{n}\right)$ with defect operator given by $D_{A_{n}}=D_{n, A}$ in $\mathcal{L}(\mathcal{X})$. Consequently, $\mathcal{D}_{A_{n}}=\mathcal{D}_{n, A}$.

The action of $A_{n}^{*}$ in the lemma is obvious by Lemma 3.1. Recall that $D_{A_{n}^{*}}=$ $\left(I-A_{n} A_{n}^{*}\right)^{1 / 2}$, where the positive square root is computed in $\mathcal{L}\left(\mathcal{X}_{n}\right)$. The formula for the action of $D_{A_{n}^{*}}$ in the lemma is obvious by the action of $A_{n}^{*}$. This completes the proof of the lemma.

Lemma 3.2 is included here for convenience; the result appeared in [16, Lemma 3.3]. Lemma 3.2 implies the following result. 
Theorem 3.2. Let $A \in \mathcal{L}(\mathcal{X})$ be an n-hypercontraction. Set $\mathcal{U}=\mathcal{D}_{A_{n}^{*}}$ and $\mathcal{Y}=\mathcal{D}_{A_{n}}=$ $\mathcal{D}_{n, A}$. Let

$$
B \in \mathcal{L}\left(\mathcal{D}_{A_{n}^{*}}, \mathcal{X}\right), \quad C \in \mathcal{L}\left(\mathcal{X}, \mathcal{D}_{n, A}\right), \quad \text { and } \quad D \in \mathcal{L}\left(\mathcal{D}_{A_{n}^{*}}, \mathcal{D}_{n, A}\right)
$$

be the operators given by

$$
B=\left.I_{n}^{-1} D_{A_{n}^{*}}\right|_{\mathcal{D}_{A_{n}^{*}}}, \quad C=D_{A_{n}}=D_{n, A}, \quad \text { and } \quad D=-\left.A_{n}^{*}\right|_{\mathcal{D}_{n}^{*}} .
$$

Then the block operator matrix

$$
\left[\begin{array}{cc}
A_{n} & B_{n} \\
C & D
\end{array}\right]=\left[\begin{array}{cc}
I_{n} A & D_{A_{n}^{*}} \\
D_{n, A} & -A_{n}^{*}
\end{array}\right]: \mathcal{X} \oplus \mathcal{D}_{A_{n}^{*}} \rightarrow \mathcal{X}_{n} \oplus \mathcal{D}_{n, A}
$$

is a unitary operator of $\mathcal{X} \oplus \mathcal{D}_{A_{n}^{*}}$ onto $\mathcal{X}_{n} \oplus \mathcal{D}_{n, A}$.

Proof. This follows from Lemma 3.2 and the preliminaries on contraction operators preceding that lemma.

Let $A \in \mathcal{L}(\mathcal{X})$ be an $n$-hypercontraction. Inspired by Theorems 3.1 and 3.2 we consider the operator-valued analytic function

$$
W_{n, A}(z)=\left.\left[-A_{n}^{*}+z D_{n, A}\left(\sum_{k=1}^{n}(I-z A)^{-k}\right) I_{n}^{-1} D_{A_{n}^{*}}\right]\right|_{\mathcal{D}_{A_{n}^{*}}}, \quad z \in \mathbb{D} .
$$

Note that the function $W_{n, A}$ is analytic in $\mathbb{D}$ because $\|A\| \leq 1$ and that $W_{n, A}(z)$ is an operator in $\mathcal{L}\left(\mathcal{D}_{A_{n}^{*}}, \mathcal{D}_{n, A}\right)$. Furthermore, if the $n$-hypercontraction $A \in \mathcal{L}(\mathcal{X})$ is in the class $C_{0}$, then by Theorem 2.1 the function $W_{n, A}$ is a Bergman inner function for $A_{n}\left(\mathcal{D}_{n, A}\right)$. Operator-valued Bergman inner functions $W_{n, A}$ of this form appear in the description of wandering subspaces in the class of vector-valued standard weighted Bergman spaces $A_{n}(\mathcal{Y})$ (see [16]), which we shall use in 84

\section{§4. Realization of Bergman inner functions}

In this section we shall show that a general operator-valued Bergman inner function can be realized as an operator-valued analytic function of the form (0.1) with $A \in \mathcal{L}(\mathcal{X})$ an $n$-hypercontraction of class $C_{0}$. and with the quadruple $(A, B, C, D)$ of operators satisfying (0.7)-(0.9).

First, we show that operator-valued Bergman inner functions naturally parameterize the wandering subspaces.

Theorem 4.1. Let $\mathcal{E}$ be a wandering subspace of $A_{n}(\mathcal{Y})$. Then there exists an $\mathcal{L}(\mathcal{U}, \mathcal{Y})$ valued Bergman inner function $W$ for $A_{n}(\mathcal{Y})$ such that

$$
\mathcal{E}=\left\{W u \in A_{n}(\mathcal{Y}): u \in \mathcal{U}\right\} .
$$

Proof. Let $\mathcal{U}=\mathcal{E}$, and for $z \in \mathbb{D}$ let $W(z) \in \mathcal{L}(\mathcal{U}, \mathcal{Y})$ be the operator defined by

$$
W(z) u=u(z), \quad u \in \mathcal{U} .
$$

We denote by $W_{k} \in \mathcal{L}(\mathcal{U}, \mathcal{Y})$ the operator defined by $W_{k} u=u^{(k)}(0) / k$ ! for $u \in \mathcal{U}(k$ th Taylor coefficient). By the expression of the norm of $A_{n}(\mathcal{Y})$, we have $\left\|W_{k}\right\|^{2} \leq 1 / \mu_{n ; k}$. A straightforward argument yields the power series expansion

$$
W(z)=\sum_{k \geq 0} W_{k} z^{k}, \quad z \in \mathbb{D} .
$$

Clearly, this shows that $W: z \mapsto W(z)$ is an $\mathcal{L}(\mathcal{U}, \mathcal{Y})$-valued analytic function in the unit disk $\mathbb{D}$. Observe that $W u=u$ for $u \in \mathcal{U}$. Properties (11) and (2) of Bergman inner functions are obvious by construction.

We record also the following uniqueness property of Bergman inner functions. 
Proposition 4.1. Let $W_{j}$ be an $\mathcal{L}\left(\mathcal{U}_{j}, \mathcal{Y}\right)$-valued Bergman inner function for $A_{n}(\mathcal{Y})(j=$ $1,2)$, and assume that $W_{1}\left(\mathcal{U}_{1}\right) \subset W_{2}\left(\mathcal{U}_{2}\right)$. Then there exists an isometry $U \in \mathcal{L}\left(\mathcal{U}_{1}, \mathcal{U}_{2}\right)$ of $\mathcal{U}_{1}$ into $\mathcal{U}_{2}$ such that

$$
W_{1}(z)=W_{2}(z) U, \quad z \in \mathbb{D} .
$$

Furthermore, the operator $U$ is uniquely determined by (4.1).

Proof. For every $u_{1} \in \mathcal{U}_{1}$ there exists $u_{2} \in \mathcal{U}_{2}$ such that $W_{1} u_{1}=W_{2} u_{2}$ in $A_{n}(\mathcal{Y})$. By property (11) of Bergman inner functions we have $\left\|u_{1}\right\|^{2}=\left\|u_{2}\right\|^{2}$. Clearly, the map $U: u_{1} \mapsto u_{2}$ gives an isometry of $\mathcal{U}_{1}$ into $\mathcal{U}_{2}$ satisfying (4.1). The uniqueness assertion in the proposition is obvious.

Next, we shall show that a general operator-valued Bergman inner function for the Bergman space $A_{n}(\mathcal{Y})$ can be realized as an operator-valued analytic function of the form (0.1) with $A \in \mathcal{L}(\mathcal{X})$ an $n$-hypercontraction of class $C_{0}$. and $(A, B, C, D)$ a quadruple of operators satisfying (0.7)-(0.9).

Theorem 4.2. Let $W$ be an $\mathcal{L}(\mathcal{U}, \mathcal{Y})$-valued Bergman inner function for $A_{n}(\mathcal{Y})$. Then there exists an $n$-hypercontraction $A \in \mathcal{L}(\mathcal{X})$ of class $C_{0}$. and operators

$$
B \in \mathcal{L}(\mathcal{U}, \mathcal{X}), \quad C \in \mathcal{L}(\mathcal{X}, \mathcal{Y}), \text { and } D \in \mathcal{L}(\mathcal{U}, \mathcal{Y})
$$

satisfying (0.7), (0.8), and (0.9) such that the function $W$ has the form (0.1).

Proof. Let $\mathcal{I}=[W(\mathcal{U})]=\bigvee_{k>0} S_{n}^{k}(W(\mathcal{U}))$ be the shift invariant subspace of $A_{n}(\mathcal{Y})$ generated by $W(\mathcal{U})$. We denote by $\mathcal{Y}^{\prime}$ the closed subspace of $\mathcal{Y}$ spanned by the values of the functions in $\mathcal{I}$, and we view $\mathcal{I}$ as a shift invariant subspace of $A_{n}\left(\mathcal{Y}^{\prime}\right)$. Set

$$
\mathcal{X}=A_{n}\left(\mathcal{Y}^{\prime}\right) \ominus \mathcal{I} \text { and } A=\left.S_{n}^{*}\right|_{\mathcal{X}} \text { in } \mathcal{L}(\mathcal{X}) \text {. }
$$

Then this operator $A \in \mathcal{L}(\mathcal{X})$ is an $n$-hypercontraction of class $C_{0}$. (see [15. Proposition 5.1]).

By [16. Theorem 5.3], there exists an isometry $V \in \mathcal{L}\left(\mathcal{D}_{n, A}, \mathcal{Y}^{\prime}\right)$ of $\mathcal{D}_{n, A}$ into $\mathcal{Y}^{\prime}$ and a unitary operator

$$
U=\left[\begin{array}{l}
U_{1} \\
U_{2}
\end{array}\right]: \mathcal{U} \rightarrow\left(\mathcal{Y}^{\prime} \ominus V\left(\mathcal{D}_{n, A}\right)\right) \oplus \mathcal{D}_{A_{n}^{*}}
$$

of $\mathcal{U}$ onto $\left(\mathcal{Y}^{\prime} \ominus V\left(\mathcal{D}_{n, A}\right)\right) \oplus \mathcal{D}_{A_{n}^{*}}$ such that the function $W$ has the form

$$
W(z)=U_{1}+V W_{n, A}(z) U_{2}, \quad z \in \mathbb{D},
$$

where the function $W_{n, A}$ is the generalized characteristic operator function for $A$ given by (0.10). We set

$$
B=I_{n}^{-1} D_{A_{n}^{*}} U_{2}, \quad C=V D_{n, A}, \quad \text { and } \quad D=U_{1}-V A_{n}^{*} U_{2} .
$$

Clearly, $B \in \mathcal{L}(\mathcal{U}, \mathcal{X})$ and $C \in \mathcal{L}(\mathcal{X}, \mathcal{Y})$. By (3.2) and Lemma 3.2 we have $A_{n}^{*}\left(\mathcal{D}_{A_{n}^{*}}\right) \subset$ $\mathcal{D}_{n, A}$. Consequently, $D \in \mathcal{L}(\mathcal{U}, \mathcal{Y})$. Obviously, (4.3) implies that with this choice of operators $(A, B, C, D)$, the function $W$ takes the form (0.1). We proceed to the proof of the fact that this quadruple $(A, B, C, D)$ of operators satisfies (0.7)-(0.9).

We verify (0.7). Since $V$ is an isometry, we have

$$
C^{*} C=D_{n, A} V^{*} V D_{n, A}=\sum_{k=0}^{n}(-1)^{k}\left(\begin{array}{l}
n \\
k
\end{array}\right) A^{* k} A^{k} \text { in } \mathcal{L}(\mathcal{X}),
$$

where the second identity follows from the definition of the defect operator $D_{n, A}$.

Now we prove (0.8). Since $V$ is an isometry, we have

$$
D^{*} C=\left(U_{1}^{*}-U_{2}^{*} A_{n} V^{*}\right) V D_{n, A}=U_{1}^{*} V D_{n, A}-U_{2}^{*} A_{n} D_{n, A} \text { in } \mathcal{L}(\mathcal{X}, \mathcal{U}) .
$$


Since $U_{1}(\mathcal{U}) \perp V\left(\mathcal{D}_{n, A}\right)$, we have $U_{1}^{*} V=0$ in $\mathcal{L}\left(\mathcal{D}_{n, A}, \mathcal{U}\right)$. We conclude that $D^{*} C=$ $-U_{2}^{*} A_{n} D_{n, A}$ in $\mathcal{L}(\mathcal{X}, \mathcal{U})$. Lemma 3.1 shows that

$$
\begin{aligned}
B^{*}\left(\sum_{k=0}^{n-1}(-1)^{k}\left(\begin{array}{c}
n \\
k+1
\end{array}\right) A^{* k} A^{k}\right) A & =U_{2}^{*} D_{A_{n}^{*}}\left(I_{n}^{-1}\right)^{*} I_{n}^{*} I_{n} A \\
& =U_{2}^{*} D_{A_{n}^{*}}\left(I_{n} I_{n}^{-1}\right)^{*} I_{n} A=U_{2}^{*} D_{A_{n}^{*}} A_{n} \text { in } \mathcal{L}(\mathcal{X}, \mathcal{U}) .
\end{aligned}
$$

Thus,

$$
D^{*} C+B^{*}\left(\sum_{k=0}^{n-1}(-1)^{k}\left(\begin{array}{c}
n \\
k+1
\end{array}\right) A^{* k} A^{k}\right) A=-U_{2}^{*} A_{n} D_{n, A}+U_{2}^{*} D_{A_{n}^{*}} A_{n}=0 \text { in } \mathcal{L}(\mathcal{X}, \mathcal{U}),
$$

where the last identity follows from (3.2).

We prove (0.9). We have

$$
\begin{aligned}
D^{*} D & =\left(U_{1}^{*}-U_{2}^{*} A_{n} V^{*}\right)\left(U_{1}-V A_{n}^{*} U_{2}\right) \\
& =U_{1}^{*} U_{1}-U_{2}^{*} A_{n} V^{*} U_{1}-U_{1}^{*} V A_{n}^{*} U_{2}+U_{2}^{*} A_{n} A_{n}^{*} U_{2}=U_{1}^{*} U_{1}+U_{2}^{*} A_{n} A_{n}^{*} U_{2} \text { in } \mathcal{L}(\mathcal{U}),
\end{aligned}
$$

where the last is true because $U_{1}^{*} V=0$ in $\mathcal{L}\left(\mathcal{D}_{n, A}, \mathcal{U}\right)$. By Lemma 3.1 we have

$$
\begin{aligned}
B^{*}\left(\sum_{k=0}^{n-1}(-1)^{k}\left(\begin{array}{c}
n \\
k+1
\end{array}\right) A^{* k} A^{k}\right) B & =U_{2}^{*} D_{A_{n}^{*}}\left(I_{n}^{-1}\right)^{*} I_{n}^{*} I_{n} I_{n}^{-1} D_{A_{n}^{*}} U_{2} \\
& =U_{2}^{*} D_{A_{n}^{*}}\left(I_{n} I_{n}^{-1}\right)^{*} D_{A_{n}^{*}} U_{2}=U_{2}^{*} D_{A_{n}^{*}}^{2} U_{2} \text { in } \mathcal{L}(\mathcal{U}) .
\end{aligned}
$$

Therefore,

$$
\begin{aligned}
D^{*} D+B^{*}\left(\sum_{k=0}^{n-1}(-1)^{k}\left(\begin{array}{c}
n \\
k+1
\end{array}\right) A^{* k} A^{k}\right) B & =U_{1}^{*} U_{1}+U_{2}^{*} A_{n} A_{n}^{*} U_{2}+U_{2}^{*} D_{A_{n}^{*}}^{2} U_{2} \\
& =U_{1}^{*} U_{1}+U_{2}^{*} U_{2}=I \text { in } \mathcal{L}(\mathcal{U}),
\end{aligned}
$$

where we have used a standard property of defect operators and the fact that $U$ is an isometry. This completes the proof of the theorem.

The above proof relies heavily on the description of a wandering subspace as presented in [16. We point out that an admissible choice of operators $(A, B, C, D)$ is given by (4.2) and (4.4) above.

\section{§5. Controllability and observability of Realizations}

In this section, we discuss the properties of approximate controllability and observability of systems of the form (0.2) with $A \in \mathcal{L}(\mathcal{X})$ an $n$-hypercontraction of class $C_{0}$. and a quadruple $(A, B, C, D)$ of operators satisfying some additional conditions related to identities (0.7)-(0.9).

Consider a system of the form (0.2). We shall refer to the subspace

$$
\mathcal{X}_{c}=\bigvee_{k \geq 0} A^{k} B(\mathcal{U})
$$

of $\mathcal{X}$ as the approximately controllable subspace of $\mathcal{X}$, and we say that system (0.2) is approximately controllable if $\mathcal{X}=\mathcal{X}_{c}$. In other words, system (0.2) is approximately controllable if and only if the subspace $B(\mathcal{U})$ is cyclic for the operator $A \in \mathcal{L}(\mathcal{X})$. Similarly, we say that the system (0.2) is approximately observable if

$$
\mathcal{X}=\bigvee_{k \geq 0} A^{* k} C^{*}(\mathcal{Y})
$$

System (0.2) is said to be minimal if it is both approximately controllable and observable. 
First, we show that systems of the form (0.2) corresponding to Bergman inner functions are always approximately observable.

Theorem 5.1. Let $W$ be an $\mathcal{L}(\mathcal{U}, \mathcal{Y})$-valued analytic function of the form (0.1) with $A \in \mathcal{L}(\mathcal{X})$ an n-hypercontraction of class $C_{0}$. and $C \in \mathcal{L}(\mathcal{X}, \mathcal{Y})$ satisfying (0.7). Then the corresponding system (0.2) is approximately observable.

Proof. System (0.2) being approximately observable amounts to saying that

$$
\mathcal{X} \ominus\left(\bigvee_{k \geq 0} A^{* k} C^{*}(\mathcal{Y})\right)=\bigcap_{k \geq 0} \operatorname{ker}\left(C A^{k}\right)=\{0\} .
$$

Assume that $C A^{k} x=0$ for $k \geq 0$. By (0.7), we have

$$
\left\|C A^{k} x\right\|^{2}=\left\langle C^{*} C A^{k} x, A^{k} x\right\rangle=\left\langle D_{n, A}^{2} A^{k} x, A^{k} x\right\rangle=\left\|D_{n, A} A^{k} x\right\|^{2},
$$

which shows that $\left\|D_{n, A} A^{k} x\right\|^{2}=0$ for $k \geq 0$. Since the operator $A \in \mathcal{L}(\mathcal{X})$ is an $n$-hypercontraction of class $C_{0}$, the operator model theory in [15, §7] says that $x=0$. This completes the proof of the theorem.

Next we discuss approximate controllability. We shall need the following lemma.

Lemma 5.1. Let $A \in \mathcal{L}(\mathcal{X})$ be an n-hypercontraction, and let $x \in \mathcal{X}$. Then $x=$ $I_{n}^{*} I_{n} A A^{*} x$ if and only if $\left\langle\left(I_{n}^{*} I_{n}\right)^{-1} x, x\right\rangle=\left\|A^{*} x\right\|^{2}$.

Proof. Assume first that $x=I_{n}^{*} I_{n} A A^{*} x$. Then $\left(I_{n}^{*} I_{n}\right)^{-1} x=A A^{*} x$, and we have

$$
\left\langle\left(I_{n}^{*} I_{n}\right)^{-1} x, x\right\rangle=\left\langle A A^{*} x, x\right\rangle=\left\|A^{*} x\right\|^{2} .
$$

This proves the "only if" part.

Next, we assume that $\left\langle\left(I_{n}^{*} I_{n}\right)^{-1} x, x\right\rangle=\left\|A^{*} x\right\|^{2}$. We proceed to the proof of the relation $x=I_{n}^{*} I_{n} A A^{*} x$. Write $x=I_{n}^{*} y$, where $y \in \mathcal{X}_{n}$. Then we have

$$
\left\langle\left(I_{n}^{*} I_{n}\right)^{-1} I_{n}^{*} y, I_{n}^{*} y\right\rangle=\left\|A^{*} I_{n}^{*} y\right\|^{2}=\left\|A_{n}^{*} y\right\|^{2},
$$

where $A_{n}=I_{n} A \in \mathcal{L}\left(\mathcal{X}, \mathcal{X}_{n}\right)$. Observe that $I_{n}^{-1}=\left(I_{n}^{*} I_{n}\right)^{-1} I_{n}^{*}$, whence

$$
\left\langle\left(I_{n}^{*} I_{n}\right)^{-1} I_{n}^{*} y, I_{n}^{*} y\right\rangle=\left\langle I_{n}^{-1} y, I_{n}^{*} y\right\rangle=\left\langle I_{n} I_{n}^{-1} y, y\right\rangle_{n}=\|y\|_{n}^{2} .
$$

We now know that $\left\|A_{n}^{*} y\right\|^{2}=\|y\|_{n}^{2}$. Recall that $A_{n}^{*} \in \mathcal{L}\left(\mathcal{X}_{n}, \mathcal{X}\right)$ is a contraction operator (see Lemma 3.2). The identity $\left\|A_{n}^{*} y\right\|^{2}=\|y\|_{n}^{2}$ means that $D_{A_{n}^{*}} y=0$, and the formula $I=A_{n} A_{n}^{*}+D_{A_{n}^{*}}^{2}$ in $\mathcal{L}\left(\mathcal{X}_{n}\right)$ implies $y=A_{n} A_{n}^{*} y$. Applying the operator $I_{n}^{*}$, we conclude that

$$
x=I_{n}^{*} y=I_{n}^{*} A_{n} A_{n}^{*} y=I_{n}^{*} I_{n} A A^{*} I_{n}^{*} y=I_{n}^{*} I_{n} A A^{*} x .
$$

This completes the proof of the lemma.

Now we describe the non-approximately-controllable subspace of $\mathcal{X}$.

Theorem 5.2. Consider a system of the form (0.2) with $A \in \mathcal{L}(\mathcal{X})$ an n-hypercontraction of class $C_{0}$. and with $B \in \mathcal{L}(\mathcal{U}, \mathcal{X})$ such that $A_{n} A_{n}^{*}+B_{n} B_{n}^{*}=I$ in $\mathcal{L}\left(\mathcal{X}_{n}\right)$. Then

$$
\begin{aligned}
\mathcal{X} \ominus \mathcal{X}_{c} & =\left\{x \in \mathcal{X}:\left\langle\left(I_{n}^{*} I_{n}\right)^{-1} A^{* k} x, A^{* k} x\right\rangle=\left\|A^{*(k+1)} x\right\|^{2} \text { for } k \geq 0\right\} \\
& =\left\{x \in \mathcal{X}: A^{* k} x=I_{n}^{*} I_{n} A A^{*(k+1)} x \text { for } k \geq 0\right\} .
\end{aligned}
$$

Proof. We have

$$
\mathcal{X} \ominus \mathcal{X}_{c}=\mathcal{X} \ominus\left(\bigvee_{k \geq 0} A^{k} B(\mathcal{U})\right)=\bigcap_{k \geq 0} \operatorname{ker}\left(B^{*} A^{* k}\right)
$$


Recall that $A_{n}=I_{n} A$ and $B_{n}=I_{n} B$. We can write

$$
B^{*} A^{* k}=B^{*} I_{n}^{*} I_{n}\left(I_{n}^{*} I_{n}\right)^{-1} A^{* k}=B_{n}^{*} I_{n}\left(I_{n}^{*} I_{n}\right)^{-1} A^{* k} \text { in } \mathcal{L}(\mathcal{X}, \mathcal{U}) .
$$

Since $A_{n} A_{n}^{*}+B_{n} B_{n}^{*}=I$ in $\mathcal{L}\left(\mathcal{X}_{n}\right)$, we see that $x \in \mathcal{X} \ominus \mathcal{X}_{c}$ if and only if

$$
\begin{aligned}
0 & =\left\|B_{n}^{*} I_{n}\left(I_{n}^{*} I_{n}\right)^{-1} A^{* k} x\right\|^{2}=\left\|I_{n}\left(I_{n}^{*} I_{n}\right)^{-1} A^{* k} x\right\|^{2}-\left\|A_{n}^{*} I_{n}\left(I_{n}^{*} I_{n}\right)^{-1} A^{* k} x\right\|^{2} \\
& =\left\langle\left(I_{n}^{*} I_{n}\right)^{-1} A^{* k} x, A^{* k} x\right\rangle-\left\|A^{*(k+1)} x\right\|^{2}
\end{aligned}
$$

for $k \geq 0$. This proves the first relation in (5.1). The second follows from Lemma 5.1

Next, we relate the non-approximately-controllable subspace to the canonical embedding of the main operator as a part of an adjoint shift. We recall (see the Introduction) the map $V_{n}$ modeling an $n$-hypercontraction $A \in \mathcal{L}(\mathcal{X})$ of class $C_{0}$. as part of the adjoint shift $S_{n}^{*}$ on $A_{n}\left(\mathcal{D}_{n, A}\right)$.

Theorem 5.3. Consider a system of the form (0.2) with $A \in \mathcal{L}(\mathcal{X})$ an n-hypercontraction of class $C_{0}$. and with $B \in \mathcal{L}(\mathcal{U}, \mathcal{X})$ such that $A_{n} A_{n}^{*}+B_{n} B_{n}^{*}=I$ in $\mathcal{L}\left(\mathcal{X}_{n}\right)$. Then

$$
\left\{x \in \mathcal{X}: S_{n}^{k}\left(V_{n} x\right) \in V_{n}(\mathcal{X}) \text { for } k \geq 1\right\} \subset \mathcal{X} \ominus \mathcal{X}_{c} .
$$

For $n=1$, equality occurs in (5.2).

Proof. We shall use the formula

$$
\left(S_{n}^{*} S_{n}\right)^{-1}=\sum_{k=0}^{n-1}(-1)^{k}\left(\begin{array}{c}
n \\
k+1
\end{array}\right) S_{n}^{k} S_{n}^{* k} \text { in } \mathcal{L}\left(A_{n}\left(\mathcal{D}_{n, A}\right)\right)
$$

for the shift operator $S_{n}$ on $A_{n}\left(\mathcal{D}_{n, A}\right)$. For a proof of this formula, we refer to 16, $\S 1$. Recall also that $V_{n} \in \mathcal{L}\left(\mathcal{X}, A_{n}\left(\mathcal{D}_{n, A}\right)\right)$ is an isometry such that $S_{n}^{*} V_{n}=V_{n} A$, and this intertwining relation yields $V_{n}^{*} S_{n}=A^{*} V_{n}^{*}$ by passage to adjoints. Performing a compression to $\mathcal{X}$, we obtain

$$
V_{n}^{*}\left(S_{n}^{*} S_{n}\right)^{-1} V_{n}=\sum_{k=0}^{n-1}(-1)^{k}\left(\begin{array}{c}
n \\
k+1
\end{array}\right) A^{* k} A^{k}=I_{n}^{*} I_{n} \text { in } \mathcal{L}(\mathcal{X}) .
$$

We shall also use the operator

$$
L_{n}=\left(S_{n}^{*} S_{n}\right)^{-1} S_{n}^{*} \text { in } \mathcal{L}\left(A_{n}\left(\mathcal{D}_{n, A}\right)\right),
$$

which is the left inverse of $S_{n}$ with $\operatorname{kernel} \operatorname{ker}\left(L_{n}\right)=\operatorname{ker}\left(S_{n}^{*}\right)$ consisting of the constant functions in $A_{n}\left(\mathcal{D}_{n, A}\right)$. The operator $L_{n}$ is realized naturally as the backward shift operator on $A_{n}\left(\mathcal{D}_{n, A}\right)$ (see Shimorin [18] for related constructions).

A computation now shows that

$$
\begin{aligned}
I_{n}^{*} I_{n} A A^{*(k+1)} & =V_{n}^{*}\left(S_{n}^{*} S_{n}\right)^{-1} V_{n} A A^{*(k+1)}=V_{n}^{*}\left(S_{n}^{*} S_{n}\right)^{-1} S_{n}^{*} V_{n} A^{*(k+1)} V_{n}^{*} V_{n} \\
& =V_{n}^{*} L_{n} V_{n} V_{n}^{*} S_{n}^{k+1} V_{n}=V_{n}^{*} P_{V_{n}(\mathcal{X})} L_{n} P_{V_{n}(\mathcal{X})} S_{n}^{k+1} V_{n} \text { in } \mathcal{L}(\mathcal{X}) .
\end{aligned}
$$

Here the last identity follows from the observation that $P_{V_{n}(\mathcal{X})}=V_{n} V_{n}^{*}$ is the orthogonal projection of $A_{n}\left(\mathcal{D}_{n, A}\right)$ onto $V_{n}(\mathcal{X})$ (see [16, Lemma 2.1]). If $x \in \mathcal{X}$ is such that $S_{n}^{k} V_{n} x \in$ $V_{n}(\mathcal{X})$ for $k \geq 1$, then

$$
\begin{aligned}
I_{n} I_{n}^{*} A A^{*(k+1)} x & =V_{n}^{*} P_{V_{n}(\mathcal{X})} L_{n} P_{V_{n}(\mathcal{X})} S_{n}^{k+1} V_{n} x=V_{n}^{*} P_{V_{n}(\mathcal{X})} L_{n} S_{n}^{k+1} V_{n} x \\
& =V_{n}^{*} P_{V_{n}(\mathcal{X})} S_{n}^{k} V_{n} x=V_{n}^{*} S_{n}^{k} V_{n} x=A^{* k} x,
\end{aligned}
$$

showing that $x \in \mathcal{X} \ominus \mathcal{X}_{c}$ by Theorem [5.2, This proves the inclusion (5.2). 
Now, let $n=1$. Observe that $I_{1}^{*} I_{1}=I$ in $\mathcal{L}(\mathcal{X})$ and that $L_{1}=\left(S_{1}^{*} S_{1}\right)^{-1} S_{1}^{*}=S_{1}^{*}$ in $\mathcal{L}\left(A_{1}\left(\mathcal{D}_{A}\right)\right)$ because $S_{1}$ is an isometry. This shows that the above formula (5.3) simplifies to

$$
A A^{*(k+1)}=V_{1}^{*} P_{V_{1}(\mathcal{X})} S_{1}^{*} P_{V_{1}(\mathcal{X})} S_{1}^{k+1} V_{1} \text { in } \mathcal{L}(\mathcal{X}) .
$$

Also by Theorem [5.2, an element $x \in \mathcal{X}$ belongs to $\mathcal{X} \ominus \mathcal{X}_{c}$ if and only if $A^{* k} x=A A^{*(k+1)} x$ for $k \geq 0$. If $x \in \mathcal{X} \ominus \mathcal{X}_{c}$, then $V_{1} A^{* k} x=V_{1} A A^{*(k+1)} x$, and formula (5.4) yields

$$
P_{V_{1}(\mathcal{X})} S_{1}^{k} V_{1} x=V_{1} A^{* k} x=P_{V_{1}(\mathcal{X})} S_{1}^{*} P_{V_{1}(\mathcal{X})} S_{1}^{k+1} V_{1} x
$$

for $k \geq 0$. For $k=0$ this means that $V_{1} x=P_{V_{1}(\mathcal{X})} S_{1}^{*} P_{V_{1}(\mathcal{X})} S_{1} V_{1} x$, and an easy estimation gives

$$
\left\|V_{1} x\right\|^{2} \leq\left\|P_{V_{1}(\mathcal{X})} S_{1} V_{1} x\right\|^{2} \leq\left\|S_{1} V_{1} x\right\|^{2}=\left\|V_{1} x\right\|^{2},
$$

showing that $S_{1} V_{1} x \in V_{1}(\mathcal{X})$. If $S_{1}^{k} V_{1} x \in V_{1}(\mathcal{X})$, we can apply the same argument to show that then also $S_{1}^{k+1} V_{1} x \in V_{1}(\mathcal{X})$. Thus, equality occurs in (5.2) if $n=1$. This completes the proof of the theorem.

We remark that the subspace

$$
\left\{f \in V_{n}(\mathcal{X}): S_{n}^{k} f \in V_{n}(\mathcal{X}) \text { for } k \geq 1\right\}
$$

of $A_{n}\left(\mathcal{D}_{n, A}\right)$ has the interpretation of being the maximal shift invariant subspace contained in $V_{n}(\mathcal{X})$. In particular, if $V_{n}(\mathcal{X})$ contains a nonzero shift invariant subspace and the operators $A \in \mathcal{L}(\mathcal{X})$ and $B \in \mathcal{L}(\mathcal{U}, \mathcal{X})$ are as in Theorem [5.3, then system (0.2) is not approximately controllable. The property of the subspace $V_{n}(\mathcal{X})$ to contain no nonzero shift invariant subspace is naturally thought of as the property of the wandering subspace for the shift invariant subspace $A_{n}\left(\mathcal{D}_{n, A}\right) \ominus V_{n}(\mathcal{X})$ to be maximal with respect to inclusion.

For $n \geq 2$, we do not know whether equality occurs in (5.2).

The assumption $A_{n} A_{n}^{*}+B_{n} B_{n}^{*}=I$ in $\mathcal{L}\left(\mathcal{X}_{n}\right)$ used in Theorems 5.2 and 5.3 is of a simplifying nature and is satisfied if the block operator matrix $\theta_{n}$ is a unitary operator of $\mathcal{X} \oplus \mathcal{U}$ onto $\mathcal{X}_{n} \oplus \mathcal{Y}$. In general, system (0.2) being approximately controllable means that the subspace $V_{n}(B(\mathcal{U}))$ of $V_{n}(\mathcal{X})$ is cyclic for the adjoint shift $S_{n}^{*}$ restricted to $V_{n}(\mathcal{X})$.

\section{§6. A multiplier property of Bergman inner FunCtions}

Bergman inner functions act naturally as contractive multipliers from the Hardy space into the Bergman space. Our purpose in this section is to give a version of this contractive multiplier property in the context of operator-valued Bergman inner functions (see Theorem 6.1) and to discuss some of its consequences.

We denote by $L_{1}$ the backward shift operator on the Hardy space $A_{1}(\mathcal{U})$, defined by

$$
\left(L_{1} u\right)(z)=\frac{u(z)-u(0)}{z}=\sum_{k \geq 0} u_{k+1} z^{k}, \quad z \in \mathbb{D}
$$

for functions $u \in A_{1}(\mathcal{U})$ of the form

$$
u(z)=\sum_{k \geq 0} u_{k} z^{k}, \quad z \in \mathbb{D} .
$$

Theorem 6.1. Let $W$ be an $\mathcal{L}(\mathcal{U}, \mathcal{Y})$-valued Bergman inner function for $A_{n}(\mathcal{Y})$ in the sense of Definition 0.1, Then

$$
\|u\|_{A_{1}}^{2}=\|W u\|_{A_{n}}^{2}+\sum_{k \geq 1}\left\|D_{S_{n}} W L_{1}^{k} u\right\|_{A_{n}}^{2}, \quad u \in A_{1}(\mathcal{U}) .
$$


In particular, the function $W$ is a contractive multiplier from the Hardy space $A_{1}(\mathcal{U})$ into the Bergman space $A_{n}(\mathcal{Y})$.

Proof. Let $u \in A_{1}(\mathcal{U})$ be a $\mathcal{U}$-valued polynomial of the form (6.1). We write the function $W u$ as

$$
W u=\sum_{k \geq 0} S_{n}^{k} W u_{k}=W u_{0}+\sum_{k \geq 1} S_{n}^{k} W u_{k} .
$$

Since in $A_{n}(\mathcal{Y})$ the element $W u_{0}$ is perpendicular to $S_{n}^{k}\left(W u_{k}\right)$ for $k \geq 1$ by property (2) of Bergman inner functions, we have

$$
\|W u\|_{A_{n}}^{2}=\left\|W u_{0}\right\|_{A_{n}}^{2}+\left\|\sum_{k \geq 1} S_{n}^{k} W u_{k}\right\|_{A_{n}}^{2}=\left\|u_{0}\right\|^{2}+\left\|S_{n}\left(\sum_{k \geq 0} S_{n}^{k} W u_{k+1}\right)\right\|_{A_{n}}^{2},
$$

where the last identity follows from property (11) of Bergman inner functions. Now we use the defect operator $D_{S_{n}}$ to rewrite the above relation as

$$
\begin{aligned}
\|W u\|_{A_{n}}^{2} & =\left\|u_{0}\right\|^{2}+\left\|\sum_{k \geq 0} S_{n}^{k} W u_{k+1}\right\|_{A_{n}}^{2}-\left\|D_{S_{n}}\left(\sum_{k \geq 0} S_{n}^{k} W u_{k+1}\right)\right\|_{A_{n}}^{2} \\
& =\left\|u_{0}\right\|^{2}+\left\|W L_{1} u\right\|_{A_{n}}^{2}-\left\|D_{S_{n}} W L_{1} u\right\|_{A_{n}}^{2} .
\end{aligned}
$$

By an induction argument, we conclude that

$$
\|W u\|_{A_{n}}^{2}=\sum_{k \geq 0}\left\|u_{k}\right\|^{2}-\sum_{k \geq 1}\left\|D_{S_{n}} W L_{1}^{k} u\right\|_{A_{n}}^{2}=\|u\|_{A_{1}}^{2}-\sum_{k \geq 1}\left\|D_{S_{n}} W L_{1}^{k} u\right\|_{A_{n}}^{2} .
$$

This proves the identity of norms in the theorem provided $u \in A_{1}(\mathcal{U})$ is a $\mathcal{U}$-valued polynomial. The case of a general function $u \in A_{1}(\mathcal{U})$ follows by a standard approximation argument. This completes the proof of the theorem.

We note that the proof of Theorem 6.1 is based on an argument of Shimorin 19 , Lemma 2.1]; see also [16, Theorem 4.1].

We shall also discuss norm bounds with respect to some more general weighted Bergman space norms generalizing the earlier notion of $A_{n}(\mathcal{U})$-spaces. We set

$$
d \mu_{\alpha}(z)=(\alpha-1)\left(1-|z|^{2}\right)^{\alpha-2} d A(z), \quad z \in \mathbb{D},
$$

where $\alpha>1$ is a real number, and

$$
\mu_{\alpha ; k}=\int_{\overline{\mathbb{D}}}|z|^{2 k} d \mu_{\alpha}(z)=\frac{k ! \Gamma(\alpha)}{\Gamma(k+\alpha)},
$$

where $k \geq 0$ is an integer; here $\Gamma(x)=\int_{0}^{\infty} t^{x-1} e^{-t} d t$ is the gamma function. If $\mathcal{U}$ is a Hilbert space and $\alpha \geq 1$ is a real number, we denote by $A_{\alpha}(\mathcal{U})$ the space of all $\mathcal{U}$-valued analytic functions

$$
u(z)=\sum_{k \geq 0} u_{k} z^{k}, \quad z \in \mathbb{D},
$$

in the unit disk $\mathbb{D}$ with finite norm

$$
\|u\|_{A_{\alpha}}^{2}=\lim _{r \rightarrow 1} \int_{\overline{\mathbb{D}}}\|u(r z)\|^{2} d \mu_{\alpha}(z)=\sum_{k \geq 0}\left\|u_{k}\right\|^{2} \mu_{\alpha ; k} .
$$

The space $A_{\alpha}(\mathcal{U})$ is a Hilbert space of $\mathcal{U}$-valued analytic functions in $\mathbb{D}$ with reproducing kernel function

$$
K_{\alpha}(z, \zeta)=\frac{1}{(1-\bar{\zeta} z)^{\alpha}} I_{\mathcal{U}}, \quad(z, \zeta) \in \mathbb{D}^{2}
$$

(see [12, Subsection 1.1] for the details).

The Shur theorem about Shur products of positive definite matrices leads to the following result on pointwise multipliers. 
Proposition 6.1. Let $\alpha, \beta \geq 1$, and let $W$ be an $\mathcal{L}(\mathcal{U}, \mathcal{Y})$-valued analytic function that is a contractive multiplier from $A_{1}(\mathcal{U})$ into $A_{\alpha}(\mathcal{Y})$. Then $W$ is a contractive multiplier from $A_{\beta}(\mathcal{U})$ into $A_{\alpha+\beta-1}(\mathcal{Y})$.

Sketch of the proof. Recall that an essential property of a kernel function is that it is positive definite. The assumption that the function $W$ is a contractive multiplier from $A_{1}(\mathcal{U})$ into $A_{\alpha}(\mathcal{Y})$ can be formulated equivalently by saying that the $\mathcal{L}(\mathcal{Y})$-valued function

$$
L(z, \zeta)=\frac{1}{(1-\bar{\zeta} z)^{\alpha}} I_{\mathcal{Y}}-\frac{1}{1-\bar{\zeta} z} W(z) W(\zeta)^{*}, \quad(z, \zeta) \in \mathbb{D}^{2},
$$

is positive definite (see, e.g., [16, $\$ 4$ ] for the details). Let $\gamma>0$, and consider the function

$$
K_{\gamma}(z, \zeta)=\frac{1}{(1-\bar{\zeta} z)^{\gamma}}=\sum_{k \geq 0} \frac{\Gamma(k+\gamma)}{k ! \Gamma(\gamma)}(\bar{\zeta} z)^{k}, \quad(z, \zeta) \in \mathbb{D}^{2} .
$$

The function $K_{\gamma}$ is positive definite, being the kernel function of a reproducing kernel Hilbert space (see also Shimorin [17] for a related discussion). A well-known result on products of reproducing kernel functions (see [6, I.8]) shows now that the function

$$
\frac{1}{(1-\bar{\zeta} z)^{\gamma}} L(z, \zeta)=\frac{1}{(1-\bar{\zeta} z)^{\alpha+\gamma}} I_{\mathcal{Y}}-\frac{1}{(1-\bar{\zeta} z)^{1+\gamma}} W(z) W(\zeta)^{*}, \quad(z, \zeta) \in \mathbb{D}^{2}
$$

is positive definite. By the same arguments as above, this means that the function $W$ is a contractive multiplier from $A_{1+\gamma}(\mathcal{U})$ into $A_{\alpha+\gamma}(\mathcal{Y})$. To arrive at the conclusion of the proposition, we set $\gamma=\beta-1$.

We list a few corollaries to Theorem 6.1.

Corollary 6.1. Let $W$ be an $\mathcal{L}(\mathcal{U}, \mathcal{Y})$-valued inner function for the Hardy space $A_{1}(\mathcal{Y})$. Then the function $W$ is an isometric multiplier of the Hardy space $A_{1}(\mathcal{U})$ into the Hardy space $A_{1}(\mathcal{Y})$.

Proof. The fact that the shift operator $S_{1}$ on $A_{1}(\mathcal{Y})$ is an isometry implies that $D_{S_{1}}=0$. The result follows from Theorem 6.1

For systems of the form (0.2) we obtain the following result.

Corollary 6.2. Let $\alpha \geq 1$ be a real number. Let $A \in \mathcal{L}(\mathcal{X})$ be an n-hypercontraction of class $C_{0}$, and let

$$
B \in \mathcal{L}(\mathcal{U}, \mathcal{X}), \quad C \in \mathcal{L}(\mathcal{X}, \mathcal{Y}), \quad \text { and } \quad D \in \mathcal{L}(\mathcal{U}, \mathcal{Y})
$$

be operators satisfying (0.7)-(0.9). If the output sequence $\left\{y_{k}\right\}_{k \geq 0}$ in $\mathcal{Y}$ is generated from the input sequence $\left\{u_{k}\right\}_{k \geq 0}$ in $\mathcal{U}$ by the system (0.2) of recurrence relations, then

$$
\sum_{k \geq 0}\left\|y_{k}\right\|^{2} \mu_{n-1+\alpha ; k} \leq \sum_{k \geq 0}\left\|u_{k}\right\|^{2} \mu_{\alpha ; k} .
$$

Proof. By Theorem 2.1, the function $W$ given by (0.1) is a Bergman inner function for $A_{n}(\mathcal{Y})$. Recall the interpretation of the function $W$ as the transfer function for the system (0.2) provided by Theorem 1.1. The claim is now an obvious consequence of Theorem6.1 and Proposition 6.1.

Remark 6.1. If $n=\alpha=1$, then equality occurs in (6.2) by Corollary 6.1

The results of Theorem 6.1 and Proposition 6.1 also provide norm bounds for inputoutput maps of cascade connections of systems of the form (0.2). Namely, let $W_{j}$ be an $\mathcal{L}\left(\mathcal{U}_{j}, \mathcal{Y}_{j}\right)$-valued Bergman inner function for $A_{n_{j}}\left(\mathcal{Y}_{j}\right)(j=1,2)$, and assume that the output space $\mathcal{Y}_{1}$ is a closed subspace of the input space $\mathcal{U}_{2}$. Then the product function

$$
\left(W_{2} W_{1}\right)(z)=W_{2}(z) W_{1}(z), \quad z \in \mathbb{D},
$$


is a contractive multiplier of $A_{1}\left(\mathcal{U}_{1}\right)$ into $A_{n_{1}+n_{2}-1}\left(\mathcal{Y}_{2}\right)$. We omit the details.

Let $A \in \mathcal{L}(\mathcal{X})$ be an $n$-hypercontraction. By Theorem 3.1, identities (0.7)-(0.9) are equivalent to the property that the block operator matrix

$$
\theta_{n}=\left[\begin{array}{cc}
A_{n} & B_{n} \\
C & D
\end{array}\right]: \mathcal{X} \oplus \mathcal{U} \rightarrow \mathcal{X}_{n} \oplus \mathcal{Y}
$$

is an isometry of $\mathcal{X} \oplus \mathcal{U}$ into $\mathcal{X}_{n} \oplus \mathcal{Y}$. For $n=1$ the latter condition means that the system matrix

$$
\theta_{1}=\left[\begin{array}{ll}
A & B \\
C & D
\end{array}\right]: \mathcal{X} \oplus \mathcal{U} \rightarrow \mathcal{X} \oplus \mathcal{Y}
$$

for system (0.3) is an isometry of $\mathcal{X} \oplus \mathcal{U}$ into $\mathcal{X} \oplus \mathcal{Y}$, which is often phrased by saying that system (0.3) is isometric.

System (0.3) is said to be contractive if the system matrix $\theta_{1}$ is a contraction operator of $\mathcal{X} \oplus \mathcal{U}$ into $\mathcal{X} \oplus \mathcal{Y}$. It is known, more generally, that for a contractive system (0.3) the transfer function attains only contractive values in the unit disk $\mathbb{D}$; that is, the transfer function for a contractive system (0.3) is a contractive multiplier from the Hardy space $A_{1}(\mathcal{U})$ into the Hardy space $A_{1}(\mathcal{Y})$ (see [7, Theorem 4.1]).

We suggest here the problem of finding a more general condition than that provided by Theorems 2.1 and 6.1 on the quadruple $(A, B, C, D)$ of operators to ensure that the function $W$ given by (0.1) is a contractive multiplier from $A_{1}(\mathcal{U})$ into $A_{n}(\mathcal{Y})$. In particular, if $A \in \mathcal{L}(\mathcal{X})$ is an $n$-hypercontraction and the block operator matrix $\theta_{n}$ is a contraction operator, does then inequality (6.2) hold true?

We mention that an $\mathcal{L}(\mathcal{U}, \mathcal{Y})$-valued analytic function $W$ in the unit disk $\mathbb{D}$ is a contractive multiplier of the Hardy space $A_{1}(\mathcal{U})$ into the Bergman space $A_{n}(\mathcal{Y})$ if and only if the function

$$
L(z, \zeta)=\frac{1}{(1-\bar{\zeta} z)^{n}} I_{\mathcal{Y}}-\frac{1}{1-\bar{\zeta} z} W(z) W(\zeta)^{*}, \quad(z, \zeta) \in \mathbb{D} \times \mathbb{D},
$$

is positive definite on $\mathbb{D} \times \mathbb{D}$. In particular, this gives the upper bound

$$
W(z) W(z)^{*} \leq \frac{1}{\left(1-|z|^{2}\right)^{n-1}} I_{\mathcal{Y}} \text { in } \mathcal{L}(\mathcal{Y}), \quad z \in \mathbb{D},
$$

for such a multiplier. We refer to [16, §4] for the details.

It is well known that an operator is a multiplier if and only if it intertwines with the shift operator.

Proposition 6.2. Let $W \in \mathcal{L}\left(A_{1}(\mathcal{U}), A_{n}(\mathcal{Y})\right)$ be a bounded linear operator. Then the intertwining relation

$$
W S_{1}=S_{n} W \quad \text { in } \quad \mathcal{L}\left(A_{1}(\mathcal{U}), A_{n}(\mathcal{Y})\right)
$$

is fulfilled if and only if $W$ is a multiplier in the sense that

$$
(W u)(z)=W(z) u(z), \quad z \in \mathbb{D}, u \in A_{1}(\mathcal{U}),
$$

for some $\mathcal{L}(\mathcal{U}, \mathcal{Y})$-valued analytic function $\mathbb{D} \ni z \mapsto W(z)$ in the unit disk.

Proof. We omit the details.

For a shift invariant subspace $\mathcal{I}$ of $A_{n}(\mathcal{Y})$, consider the operator

$$
L_{\mathcal{I}}=\left(\left.\left(\left.S_{n}\right|_{\mathcal{I}}\right)^{*} S_{n}\right|_{\mathcal{I}}\right)^{-1}\left(\left.S_{n}\right|_{\mathcal{I}}\right)^{*} \text { in } \mathcal{L}(\mathcal{I}) .
$$

The operator $L_{\mathcal{I}}$ is the left inverse of $\left.S_{n}\right|_{\mathcal{I}}$ with kernel $\mathcal{E}_{\mathcal{I}}$ :

$$
\left.L_{\mathcal{I}} S_{n}\right|_{\mathcal{I}}=I \text { in } \mathcal{L}(\mathcal{I}) \text { and } \operatorname{ker}\left(L_{\mathcal{I}}\right)=\operatorname{ker}\left(\left(\left.S_{n}\right|_{\mathcal{I}}\right)^{*}\right)=\mathcal{E}_{\mathcal{I}} \text {, }
$$

where $\mathcal{E}_{\mathcal{I}}=\mathcal{I} \ominus S_{n}(\mathcal{I})$ is the wandering subspace for $\mathcal{I}$. 
Intertwining with the operator $L_{\mathcal{I}}$ is related to property (2) of Bergman inner functions as follows.

Proposition 6.3. Let $\mathcal{I}$ be a shift invariant subspace of $A_{n}(\mathcal{Y})$, and let $W \in \mathcal{L}\left(A_{1}(\mathcal{U}), \mathcal{I}\right)$ be an operator such that $S_{n} W=W S_{1}$. Then the intertwining relation

$$
L_{\mathcal{I}} W=W L_{1} \quad \text { in } \quad \mathcal{L}\left(A_{1}(\mathcal{U}), \mathcal{I}\right)
$$

is fulfilled if and only if

$$
W(\mathcal{U})=\{W u: u \in \mathcal{U}\} \subset \mathcal{E}_{\mathcal{I}},
$$

where $\mathcal{E}_{\mathcal{I}}$ is the wandering subspace for $\mathcal{I}$.

Proof. Assume that (6.3) is true. Then for $u \in \mathcal{U}$ we have $L_{\mathcal{I}} W u=W L_{1} u=0$ in $\mathcal{I}$, which implies that $W u \in \operatorname{ker}\left(L_{\mathcal{I}}\right)=\mathcal{E}_{\mathcal{I}}$. Thus, $W(\mathcal{U}) \subset \mathcal{E}_{\mathcal{I}}$.

Now, assume that $W(\mathcal{U}) \subset \mathcal{E}_{\mathcal{I}}$. We proceed to the verification of (6.3). Let $u \in A_{1}(\mathcal{U})$. We write this function as $u=u_{0}+S_{1} L_{1} u$ with $u_{0} \in \mathcal{U}$. We have

$$
L_{\mathcal{I}} W u=L_{\mathcal{I}} W u_{0}+L_{\mathcal{I}} W S_{1} L_{1} u=L_{\mathcal{I}} W u_{0}+L_{\mathcal{I}} S_{n} W L_{1} u,
$$

where the last identity is true because $S_{n} W=W S_{1}$. Observe that $L_{\mathcal{I}} W u_{0}=0$ because $W(\mathcal{U}) \subset \mathcal{E}_{\mathcal{I}}$, and that $L_{\mathcal{I}} S_{n} W L_{1} u=W L_{1} u$ because $L_{\mathcal{I}}$ is a left inverse of $\left.S_{n}\right|_{\mathcal{I}}$. We conclude that $L_{\mathcal{I}} W u=W L_{1} u$. This completes the proof of the proposition.

We mention that operators of the form $L_{\mathcal{I}}$ above play an important role in the analysis of approximation theorems of the so-called wandering subspace type (see [14, 18]).

\section{REFERENCES}

[1] J. Agler, The Arveson extension theorem and coanalytic models, Integral Equations Operator Theory 5 (1982), 608-631. MR0697007 (84g:47011)

[2] Hypercontractions and subnormality, J. Operator Theory 13 (1985), 203-217. MR0775993 (86i:47028)

[3] A. Aleman, S. Richter, and C. Sundberg, Beurling's theorem for the Bergman space, Acta Math. 177 (1996), 275-310. MR 1440934 (98a:46034)

[4] C.-G. Ambrozie, M. Engliš, and V. Müller, Operator tuples and analytic models over general domains in $\mathbb{C}^{n}$, J. Operator Theory 47 (2002), 287-302. MR1911848(2004c:47013)

[5] J. Arazy and M. Englišs, Analytic models for commuting operator tuples on bounded symmetric domains, Trans. Amer. Math. Soc. 355 (2003), 837-864. MR1932728 (2003k:47019)

[6] N. Aronszajn, Theory of reproducing kernels, Trans. Amer. Math. Soc. 68 (1950), 337-404. MR0051437(14:479c)

[7] J. A. Ball and N. Cohen, de Branges-Rovnyak operator models and systems theory: A survey, Topics in Matrix and Operator Theory (Rotterdam, 1989), Oper. Theory Adv. Appl., vol. 50, Birkhäuser, Basel, 1991, pp. 93-136. MR 1115026 (93a:47011)

[8] I. Gohberg, S. Goldberg, and M. A. Kaashoek, Classes of linear operators. Vol. II, Oper. Theory Adv. Appl., vol. 63, Birkhäuser, Basel, 1993. MR1246332 (95a:47001)

[9] P. R. Halmos, Normal dilations and extensions of operators, Summa Brasil. Math. 2 (1950), 125134. MR0044036(13:359b)

[10] _ Shifts on Hilbert spaces, J. Reine Angew. Math. 208 (1961), 102-112. MR0152896 (27:2868)

[11] H. Hedenmalm, A factorization theorem for square area-integrable analytic functions, J. Reine Angew. Math. 422 (1991), 45-68. MR.1133317 (93c:30053)

[12] H. Hedenmalm, B. Korenblum, and K. Zhu, Theory of Bergman spaces, Grad. Texts in Math., vol. 199, Springer-Verlag, New York, 2000. MR1758653 (2001c:46043)

[13] J. W. Helton, Discrete time systems, operator models, and scattering theory, J. Funct. Anal. 16 (1974), 15-38. MR0445310 (56:3652)

[14] A. Olofsson, Wandering subspace theorems, Integral Equations Operator Theory 51 (2005), 395409. MR2126818 (2005k:47019)

[15] __ An operator-valued Berezin transform and the class of n-hypercontractions, Integral Equations Operator Theory 58 (2007), no. 4, 503-549. http://www.math.kth.se/ ^ao/. MR2329133

[16] $ـ$ A characteristic operator function for the class of n-hypercontractions, J. Funct. Anal. 236 (2006), 517-545. MR2240173(2007e:47018) 
[17] S. M. Shimorin, Double power series and reproducing kernels, Complex Analysis, Operators, and Related Topics, Oper. Theory Adv. Appl., vol. 113, Birkhäuser, Basel, 2000, pp. 339-348. MR1771773 (2001d:46042)

[18] - Wold-type decompositions and wandering subspaces for operators close to isometries, J. Reine Angew. Math. 531 (2001), 147-189. MR:1810120 (2002c:47018)

[19] - On Beurling-type theorems in weighted $l^{2}$ and Bergman spaces, Proc. Amer. Math. Soc. 131 (2003), 1777-1787. MR 1955265(2004a:47008)

[20] B. Sz.-Nagy and C. Foiaş, Harmonic analysis of operators on Hilbert space, North-Holland, New York, 1970. MR0275190(43:947)

Falugatan 22 1Tr, SE-113 32 Stockholm, Sweden

E-mail address: ao@math.kth.se

Received 4/SEP/2006

Originally published in English 